\title{
Review \\ The Effects of Nutritional Interventions on the Cognitive Development of Preschool-Age Children: A Systematic Review
}

\author{
Marina Roberts ${ }^{1}$, Terezie Tolar-Peterson ${ }^{1, *(1)}$, Abby Reynolds ${ }^{1}$, Caitlin Wall ${ }^{1}$, Nicole Reeder ${ }^{1}$ \\ and Gina Rico Mendez ${ }^{2}$ \\ 1 Department of Food Science, Nutrition and Health Promotion, Mississippi State University, \\ Starkville, MS 39762, USA; mr2447@msstate.edu (M.R.); amr503@msstate.edu (A.R.); \\ cw2693@msstate.edu (C.W.); nr657@msstate.edu (N.R.) \\ 2 Social Science Research Center, Mississippi State University, Starkville, MS 39762, USA; \\ gina.mendez@ssrc.msstate.edu \\ * Correspondence: terezie.mosby@msstate.edu
}

Citation: Roberts, M.; Tolar-Peterson, T.; Reynolds, A.; Wall, C.; Reeder, N.; Rico Mendez, G. The Effects of Nutritional Interventions on the Cognitive Development of Preschool-Age Children: A Systematic Review. Nutrients 2022, 14, 532.

https://doi.org/10.3390/ nu14030532

Academic Editors: Silvia Scaglioni,

Alessandra Mazzocchi and

Valentina De Cosmi

Received: 19 December 2021

Accepted: 21 January 2022

Published: 26 January 2022

Publisher's Note: MDPI stays neutral with regard to jurisdictional claims in published maps and institutional affiliations.

Copyright: (C) 2022 by the authors. Licensee MDPI, Basel, Switzerland. This article is an open access article distributed under the terms and conditions of the Creative Commons Attribution (CC BY) license (https:// creativecommons.org/licenses/by/ $4.0 /)$

Abstract: The developing human brain requires all essential nutrients to form and to maintain its structure. Infant and child cognitive development is dependent on adequate nutrition. Children who do not receive sufficient nutrition are at high risk of exhibiting impaired cognitive skills. This systematic review aimed to examine the effects of nutritional interventions on cognitive outcomes of preschool-age children. PubMed, PsycInfo, Academic Search Complete, and Cochrane Library electronic databases were searched to identify Randomized Controlled Trials (RCTs) published after the year 2000. Studies assessing the effects of food-based, single, and multiple micronutrient interventions on the cognition of nourished and undernourished children aged 2-6 years were deemed eligible. A total of 12 trials were identified. Eight out of the twelve studies found significant positive effects on cognitive outcomes. Iron and multiple-micronutrients supplementation yield improvements in the cognitive abilities of undernourished preschool-age children. Increased fish consumption was found to have a beneficial effect in the cognitive outcomes of nourished children. On the other hand, B-vitamin, iodized salt, and guava powder interventions failed to display significant results. Findings of this review highlight the importance of adequate nutrition during preschool years, and the crucial role sufficient nutrition plays in cognitive development.

Keywords: child development; cognition; preschool; nutrition

\section{Introduction}

\subsection{Nutrients and Cognitive Development}

Malnutrition is characterized by an imbalance between a person's nutrient requirements and their nutrient consumption, and includes conditions of overnutrition and undernutrition [1-3]. Undernutrition is caused by an inadequate intake of energy, protein, or vitamins and minerals [2], and is a present-day global problem hindering the development of young children [4-9]. For young children, undernutrition can cause emaciation, stunting, and wasting, or various micronutrient deficiencies [1-4]. Worldwide, 149 million children are stunted and 45 million are wasted [4]. Inadequate protein and energy intake in childhood is directly associated with reduced growth, and is indicative of several psychosocial problems later in life $[3,10]$. Undernourished children also exhibit impaired development and decreased functional capacity [10]. Pediatric undernutrition is characterized by a lack of adequate weight gain, low weight per height, or low weight per length, and is a direct contributor to impaired cognitive skills [11-13].

The human brain requires all essential nutrients, including protein, fats, carbohydrates, vitamins, minerals, and water, to form and maintain its structure. Therefore, adequate nutrition is essential for brain development and function [14-16]. However, micronutrients, such as iron, zinc, choline, iodine, folate, B12, and long-chain polyunsaturated fatty 
acids (LC-PUFAs) have been identified to be particularly relevant to cognitive development [14]. Iron is essential for the development of neurological pathways in the brain that influence brain function [15,17-19]. During the first two years of life, children experience rapid growth, which increases their iron requirement and places them at a higher risk for iron deficiency anemia [20]. Iron deficiency or iron deficiency anemia can negatively impact overall intelligence and cognitive development, especially if it occurs in early childhood $[15,19,21]$. Zinc is an essential trace mineral present in the brain that contributes to cerebral structure and function [22]. Zinc deficiency during infancy is associated with motor development delays [23] and detrimental effects on attention and short-term memory [15]. Long-term zinc deficiency is associated with stunting [24,25]. Choline is essential for the structural integrity of cell membranes and myelination [9,26,27]. Animal studies have shown choline deficiency to adversely impact memory $[15,28,29]$. Yet, the effects of choline on cognition in humans are still not fully understood. Iodine is an essential mineral for thyroid hormone synthesis and is required for brain development [15]. Iodine deficiency can have detrimental effects on cognitive function, and is the primary cause of intellectual disability around the world $[15,30]$. Folate is a water-soluble vitamin needed for DNA and RNA synthesis and the formation of the nervous system [15,31,32]. Maternal folate deficiency during the early stages of pregnancy is associated with an increased incidence of congenital malformations, including spina bifida and anencephaly [15,33]. Vitamin B12 is a cofactor in numerous catalytic reactions required for neurotransmitter synthesis and functioning [31,34]. Studies have linked B12 deficiency to cerebral atrophy and neurological disorders [34,35]. Vitamin A plays a critical-essential role in visual function [14,15]. LC-PUFAs, specifically docosahexaenoic acid (DHA) and eicosapentaenoic acid (EPA), are required for brain growth and development [15]. Inadequate intake of LC-PFAs is associated with impaired neurodevelopment, visual recognition, and memory [36-38].

\subsection{Nutritional Interventions during the Preschool Years and Cognitive Outcomes}

The first 1000 days of life are a crucial brain development period in which adequate nutrition is vital for optimal growth and cognitive development [39-41]. This has been identified as a sensitive time in which children are most vulnerable to behavioral and cognitive deficits [39]. A systematic review focusing on the first 1000 days of life identified the important role macronutrients, such as protein and LC-PUFAs, play in optimizing brain development [7]. Specifically, protein-energy malnutrition in early life can impede adequate brain growth, resulting in smaller brains [7]. Another review showed maintenance of adequate iron and zinc status contributes to adequate growth in early life, as significant positive effects were seen on child weight-for-age $z$-score (WAZ) and weight-for-height $z$-score [42]. Furthermore, maternal or child supplementation with choline has also been shown to support normal brain development [43]. Since the identification of the first 1000 days of life as a crucial cognitive development period, policy makers have placed strong emphasis on implementing nutritional policies that promote the healthy brain development of infants and toddlers [7]. However, public policy often does not extend to preschool-age children, even though the second 1000 days of life also represent a critical time in children's cognitive and behavioral growth [44]. Children experience the most dynamic developmental changes during the preschool years, and acquire important skills that contribute to school readiness [44]. In particular, working memory and attention control undergo rapid progress, having an extensive impact on children's academic achievement in later years $[44,45]$. Cognitive development reached in preschool years often predicts later achievements in life [45-47]. However, few articles have explored the effects of nutritional interventions on the cognitive outcomes of preschool-aged children. Children who do not receive adequate nutrition and psychosocial stimulation are likely to underperform in school and to have poor levels of cognition and education, which are linked to low-income earnings later in life $[46,48,49]$. This systematic review aimed to synthesize and evaluate the impact of nutritional interventions on the cognitive outcomes of preschool-aged children. The effects of food-based, single, and multiple micronutrient supplementation interventions 
were considered, in order to explore the correlation between nutrition interventions and cognitive performance.

\section{Methods}

This systematic search of scientific literature was conducted in May 2021, following the Preferred Reporting Items for Systematic Reviews and Meta-Analyses (PRISMA) guidelines [50]. The search strategy was developed in collaboration with an agriculture and forest resources librarian and with the guidance of the Population, Intervention, Comparators, Outcome (PICO) framework [51,52] (Table A1). Figure 1 illustrates the PRISMA flow diagram portraying the stream of evidence in different phases of this review.

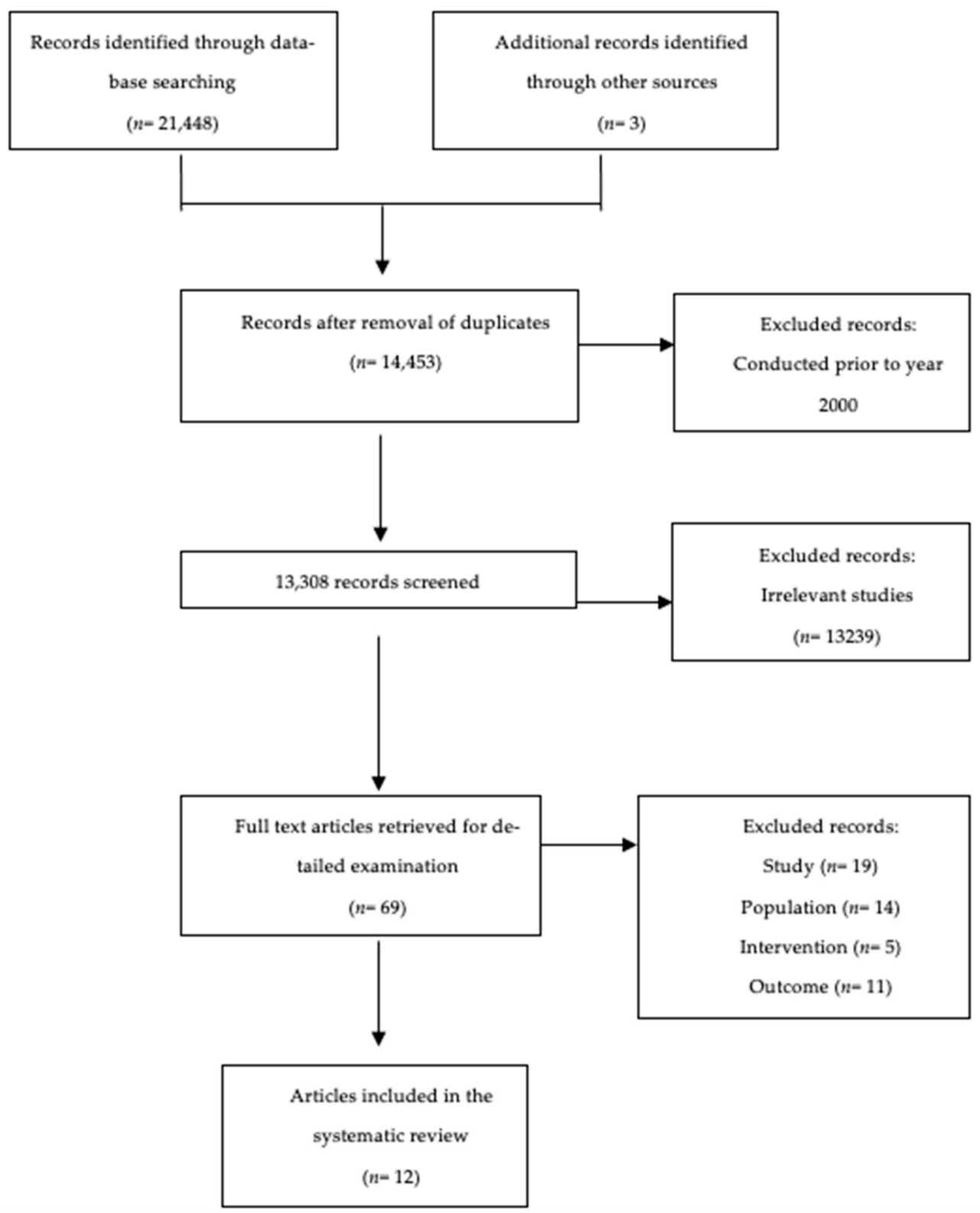

Figure 1. Study Selection Flow Diagram.

\subsection{Search Strategy}

The authors used PubMed, PsycInfo, Academic Search Complete, and Cochrane Library electronic databases. RCTs performed between 2000 and 2021 were retrieved. The following search terms were used: (nutrient* OR nutrition* OR micronutrient* OR macronutrient* OR diet* OR "meal diversity" OR "food intake") AND ("child development" 
OR cognition OR focus OR brain OR attentiveness OR attention OR memory OR verbal OR vocabulary OR learning OR literacy OR neuro* OR problem-solving OR reasoning OR "school performance" OR school) AND (child* OR preschool* OR "school age" OR school-age). Search terms were adjusted for each database to optimize the record retrieval process (Table A2) with the guidance of the Cochrane highly sensitive search strategies for identifying randomized trials [53].

\subsection{Data Extraction}

One author worked autonomously to determine the eligibility of titles and abstracts retrieved by the initial computerized search. Two other authors worked independently to evaluate qualified full-text records. Eligibility conflicts between reviewers were solved through discussions and with the assistance of a fourth reviewer. A backward search was conducted, in which reference pages of eligible articles were reviewed to ensure no pertinent studies were ignored in this review. Inclusion criteria consisted of: RCTs conducted after the year 2000, and that assessed cognitive outcomes of subjects aged 2-6 years consequent to food fortification, supplementation, or food-based interventions. Trials featuring secondary interventions including psychosocial stimulation and anthelmintic treatment combined with nutritional interventions were deemed eligible if cognitive function was a designated primary outcome. No language, geographical, or study duration restrictions were imposed. Exclusion criteria comprised: cross-sectional studies, non-randomized controlled trials, small sample size RCTs (<60 subjects), trials conducted in disease-specific population, studies focusing on children older than 6 years or younger than 2 years of age, trials providing dietary interventions targeted to the first 1000 days of life, and those that only provided parent nutrition education as the nutritional intervention. Table 1 includes a detailed description of the inclusion and exclusion criteria.

Table 1. Inclusion and Exclusion Criteria.

\begin{tabular}{|c|c|c|c|c|}
\hline Criteria & Study Design & Population & Intervention & Outcome \\
\hline \multirow[t]{3}{*}{ Include } & $\begin{array}{c}\text { RCTs } \\
\text { conducted after the year } 2000\end{array}$ & $\begin{array}{l}\text { Preschool Children } \\
\text { ( } 2-6 \text { years of age) }\end{array}$ & $\begin{array}{l}\text { Nutritional intervention } \\
\text { including food-based, } \\
\text { single, and multiple } \\
\text { micronutrient } \\
\text { supplementation } \\
\text { intervention/s }\end{array}$ & $\begin{array}{l}\text { Cognitive outcomes measured using } \\
\text { cognitive assessment tests }\end{array}$ \\
\hline & & $\begin{array}{l}\text { Healthy children and } \\
\text { children suffering from } \\
\text { undernutrition, anemia, } \\
\text { parasitic infections, or HIV }\end{array}$ & $\begin{array}{l}\text { Nutritional intervention/s } \\
\text { provided after the first } \\
\text { 1000days and in children } \\
<6 \text { years of age }\end{array}$ & $\begin{array}{c}\text { Cognition was measured after the } \\
\text { first } 1000 \text { days or in children }<6 \\
\text { years of age }\end{array}$ \\
\hline & $\begin{array}{c}\text { All other study designs and } \\
\text { animal studies } \\
\text { RCTs with a sample size }<60 \text { subjects }\end{array}$ & $\begin{array}{l}\text { Newborns, infants, primary } \\
\text { school-aged children, } \\
\text { adolescents, adults, elderly }\end{array}$ & $\begin{array}{l}\text { Nutritional intervention/s } \\
\text { not provided to } \\
\text { preschool-aged children }\end{array}$ & $\begin{array}{l}\text { Cognitive outcomes not measured in } \\
\text { preschool-aged children }\end{array}$ \\
\hline Exclude & & $\begin{array}{l}\text { Children with specific } \\
\text { diseases, such as cystic } \\
\text { fibrosis, attention deficit } \\
\text { hyperactivity disorder } \\
\text { (ADHD), epilepsy, } \\
\text { phenylketonuria, autism, } \\
\text { and gluten-related } \\
\text { neurological disorders }\end{array}$ & & \\
\hline
\end{tabular}

\subsection{Risk of Bias}

Retrieved studies were assessed by two reviewers independently using the Quality Criteria Checklist (QCC) for Primary Research from the American Dietetic Association [54]. The risk of bias tool includes four questions on relevance and ten questions on validity to appraise the appropriateness of study designs and the quality of how the studies were conducted [54]. The items assessed by the QCC include the research question, subject selection, comparability of groups, withdrawals, blinding, intervention/exposure, outcomes, analysis, conclusion support, and the likelihood of bias. Each item was classified as "Yes", "No", or "Unclear." Studies were classified as negative $(-)$ if six or more validity questions were answered as "No." A positive $(+)$ sign was assigned if the first four items and most 
answers were "Yes." If the answer to any of the first four items was "No" and other items indicated strengths, the study was classified as neutral (Ø).

\section{Results}

\subsection{Selection of Studies}

The comprehensive database search resulted in 14,453 records once duplicates were removed. After reviewing titles and abstracts, 13,239 irrelevant studies were removed. A total of 69 full-text articles were further assessed. This systematic review identified 12 RCTs that met the inclusion criteria, of which three articles were identified through a backward search of the reference list of included publications. All selected studies were published in English.

Due to the high heterogeneity among studies, a meta-analysis was not deemed appropriate for this review. Specifically, variations in the way cognitive outcomes were defined and measured, as well as differences in the type and length of nutritional interventions, created major interpretative challenges.

\subsection{Description of Studies}

Table 2 summarizes the characteristics of RCTs contained in this review. In summary, $50 \%$ of trials were conducted in developed countries [55-60] and 50\% in low and middleincome countries (LMICs) [61-66]. Four experimental studies were conducted in rural areas with low socioeconomic status $[61,62,64,66]$, and six studies in urban areas with high socioeconomic status [55-60]. The earliest trial was completed in 2004 [58] and the latest in 2020 [65]. The shortest intervention was implemented for two months [58], and the longest trial lasted ten months [61].

Table 2. Overview of the twelve RCTs exploring the effects of nutritional interventions on the cognitive development of preschool age children.

\begin{tabular}{|c|c|c|c|c|c|c|c|c|c|}
\hline $\begin{array}{l}\text { Reference, Year, } \\
\text { Country }\end{array}$ & $\begin{array}{l}\text { Sample } \\
\text { Size }\end{array}$ & Age & $\begin{array}{l}\text { Subject Characteristics } \\
\text { at Baseline }\end{array}$ & Intervention Group/s & Control Group/s & Duration & Cognitive Tests & $\begin{array}{l}\text { Cognitive Domain } \\
\text { Assessed }\end{array}$ & $\begin{array}{l}\text { Major Cognitive } \\
\text { Outcomes }\end{array}$ \\
\hline $\begin{array}{l}\text { Rauh-Pfeiffer et al. } \\
\text { [55], 2014, } \\
\text { Germany }\end{array}$ & 250 & $4-6 y$ & $\begin{array}{l}\text { Urban area, high } \\
\text { socioeconomic status, } \\
\text { healthy children with } \\
\text { low but not insuficient } \\
\text { total folate catabolite } \\
\text { concentrations }(<34 \\
\mathrm{nmol} / \mathrm{mmol} \text { creatinine) }\end{array}$ & $\begin{array}{l}\text { Children received } \\
\text { flavorless powder } \\
\text { containing folic acid } \\
(220 \mu \mathrm{g}) \text {, riboflavin }(1.1 \\
\mathrm{mg}) \text {, pyridoxine }(0.73 \\
\mathrm{mg}) \text {, cobalamin }(1.2 \mu \mathrm{\mu g}) \\
\text { and calcium lactate } \\
\text { pentahydrate }(130 \mathrm{mg})\end{array}$ & $\begin{array}{l}\text { Children received } \\
\text { flavorless powder } \\
\text { in sachets } \\
\text { matching the } \\
\text { intervention } \\
\text { product in taste } \\
\text { and appearance } \\
\text { containing only } \\
130 \mathrm{mg} \text { of calcium }\end{array}$ & 3 months & $\begin{array}{l}\text { WPPSI-III } \\
\& \text { (K-ABC) }\end{array}$ & $\begin{array}{l}\text { Verbal I.Q., short-term } \\
\text { memory, and processing } \\
\text { speed }\end{array}$ & $\begin{array}{l}\text { No significant difference } \\
\text { between groups }\end{array}$ \\
\hline $\begin{array}{l}\text { Aboud et al. [61], } \\
\text { 2017, Ethiopia }\end{array}$ & 1602 & $4-6 y$ & $\begin{array}{c}\text { Rural, low } \\
\text { socioeconomic status, } \\
\text { two control and four } \\
\text { intervention districts } \\
\text { had high UIC levels at } \\
\text { baseline }\end{array}$ & $\begin{array}{l}\text { Children had access to } \\
\text { iodized salt for } 8 \text { to } \\
10 \text { months. Children } \\
\text { received iodized salt via } \\
\text { assistance from regular } \\
\text { salt distributors }\end{array}$ & $\begin{array}{l}\text { Children had } \\
\text { access to } \\
\text { non-iodized salt } \\
\text { for } 4 \text { months, and } \\
4 \text { to } 6 \text { months of } \\
\text { iodized salt. } \\
\text { Iodized salt was } \\
\text { introduced by } \\
\text { market forces }\end{array}$ & 10 months & WPPSI & $\begin{array}{l}\text { Verbal and nonverbal } \\
\text { reasoning, and school } \\
\text { readiness }\end{array}$ & $\begin{array}{l}\text { No significant difference } \\
\text { between groups }\end{array}$ \\
\hline $\begin{array}{c}\text { Demmelmair et al. } \\
\text { [56], 2019, } \\
\text { Germany }\end{array}$ & 205 & $4-6 y$ & $\begin{array}{l}\text { Urban area, high } \\
\text { socioeconomic status, } \\
\text { healthy children }\end{array}$ & $\begin{array}{l}\text { Children received three } \\
\text { meals weekly containing } \\
50 \mathrm{~g} \text { Atlantic salmon per } \\
\text { meal }\end{array}$ & $\begin{array}{c}\text { Children received } \\
\text { three meals } \\
\text { weekly containing } \\
50 \mathrm{~g} \text { of meat per } \\
\text { meal }\end{array}$ & 4 months & $\underset{\text { 9-HPT }}{\text { WPPSI-III \& }}$ & $\begin{array}{l}\text { Fine-motor skills, verbal } \\
\text { reasoning, vocabulary, } \\
\text { word and matrix } \\
\text { reasoning, picture } \\
\text { concepts, processing } \\
\text { speed, coding, and } \\
\text { symbol search }\end{array}$ & $\begin{array}{l}\text { Intervention children } \\
\text { displayed superior } \\
\text { outcomes in WPPSI-III } \\
\text { FIQ and PIQ. No } \\
\text { significant changes were } \\
\text { found in the WPPSI-III } \\
\text { I.Q. scale scores between } \\
\text { groups }\end{array}$ \\
\hline $\begin{array}{l}\text { Choudhury et al. } \\
\text { [62], 2021, India }\end{array}$ & 352 & $3-5 y$ & $\begin{array}{c}\text { Rural, low } \\
\text { socioeconomic status, } \\
\text { ICDS beneficiaries only, } \\
\text { and children with } \\
\text { hemoglobin } \\
\text { concentration }>7 \mathrm{~g} / \mathrm{dL}\end{array}$ & $\begin{array}{l}\text { Children received } 25 \mathrm{~g} \\
\text { of guava with a } \\
\text { supplementary meal } \\
\text { (guava group) or } 25 \mathrm{~g} \text { of } \\
\text { banana with a meal } \\
\text { (banana group) }\end{array}$ & $\begin{array}{l}\text { Children did not } \\
\text { receive any fruits } \\
\text { with the meal } \\
\text { (cucumber was } \\
\text { given with meal if } \\
\text { caregivers of } \\
\text { participants } \\
\text { wished) }\end{array}$ & 8 months & MSEL & $\begin{array}{l}\text { Visual reception, } \\
\text { expressive language } \\
\text { development, and } \\
\text { fine-motor coordination }\end{array}$ & $\begin{array}{l}\text { No significant difference } \\
\text { between groups }\end{array}$ \\
\hline
\end{tabular}


Table 2. Cont.

\begin{tabular}{|c|c|c|c|c|c|c|c|c|c|}
\hline $\begin{array}{l}\text { Reference, Year, } \\
\text { Country }\end{array}$ & $\begin{array}{l}\text { Sample } \\
\text { Size }\end{array}$ & Age & $\begin{array}{c}\text { Subject Characteristics } \\
\text { at Baseline }\end{array}$ & Intervention Group/s & Control Group/s & Duration & Cognitive Tests & $\begin{array}{c}\text { Cognitive Domain } \\
\text { Assessed }\end{array}$ & $\begin{array}{l}\text { Major Cognitive } \\
\text { Outcomes }\end{array}$ \\
\hline $\begin{array}{l}\text { Øyen et al. [57], } \\
\text { 2018, Norway }\end{array}$ & 232 & $4-6 y$ & $\begin{array}{l}\text { Urban area, high } \\
\text { socioeconomic status, } \\
\text { healthy children }\end{array}$ & $\begin{array}{l}\text { Children received three } \\
\text { lunch meals per week } \\
\text { with fatty fish } \\
\text { (herring/mackerel), } \\
\text { with a mean (SD) of } 15.2 \\
\text { (14.2) } \mathrm{mg} / \mathrm{g} \text { EPA + DHA }\end{array}$ & $\begin{array}{c}\text { Children received } \\
\text { three lunch meals } \\
\text { per week with } \\
\text { meat } \\
\text { (chicken/lamb/beef) } \\
\text { with mean (SD) of } \\
0.21(0.15) \mathrm{mg} / \mathrm{g} \\
\text { EPA + DHA }\end{array}$ & 4 months & $\begin{array}{l}\text { WPPSI-III \& } \\
\text { 9-HPT }\end{array}$ & $\begin{array}{l}\text { Fine-motor skills, verbal } \\
\text { reasoning, vocabulary, } \\
\text { word and matrix } \\
\text { reasoning, picture } \\
\text { concepts, processing } \\
\text { speed, coding, and } \\
\text { symbol search }\end{array}$ & $\begin{array}{l}\text { Intervention children } \\
\text { improved speed of } \\
\text { processing and } \\
\text { fine-motor coordination } \\
\text { in a sub-analysis } \\
\text { adjusting for dietary } \\
\text { compliance. No } \\
\text { significant difference } \\
\text { was found between in } \\
\text { main analysis of total } \\
\text { I.Q. scores (WPPSI-III) }\end{array}$ \\
\hline $\begin{array}{l}\text { Schneider et al. } \\
\text { [63], 2018, } \\
\text { Indonesia }\end{array}$ & 192 & $3-5 y$ & $\begin{array}{l}\text { Urban, an upper } \\
\text { middle-income country, } \\
\text { children with a } \\
\text { below-average level of } \\
\text { stimulation at home, } \\
\text { normal cognitive } \\
\text { development, and } \\
\text { weight for height within } \\
\text { 2SD from the median } \\
\text { z-score }\end{array}$ & $\begin{array}{c}\text { Children consumed milk } \\
\text { powder (477.7kcal) } \\
\text { fortified with zinc (8), } \\
\text { iron (11.4), magnesium } \\
\text { (141), thiamin (1), niacin } \\
\text { (11), pyridoxine (1.7), } \\
\text { biotin (0.0177), Vitamin } \\
\text { C (97.3), AHA (556.6) } \\
\text { mg/100 g, and } \\
\text { performed psychosocial } \\
\text { stimulation } 3 \text { times a } \\
\text { week }\end{array}$ & $\begin{array}{c}\text { Children } \\
\text { consumed } 72 \mathrm{~g} \text { of } \\
\text { unfortified } \\
\text { skimmed milk } \\
\text { powder diluted in } \\
180 \mathrm{~mL} \text { of warm } \\
\text { water (467.8 kcal) } \\
\text { and did not } \\
\text { receive } \\
\text { psychosocial } \\
\text { stimulation }\end{array}$ & 6 months & $\begin{array}{l}\text { WPPSI-IV, CBCL } \\
1.5-5 \& \text { PICCOLO }\end{array}$ & $\begin{array}{l}\text { Cognitive functioning, } \\
\text { cognitive development, } \\
\text { memory, language, } \\
\text { psychomotor skills, } \\
\text { problem-solving, and } \\
\text { attention }\end{array}$ & $\begin{array}{l}\text { Children in the } \\
\text { intervention group } \\
\text { displayed increased } \\
\text { cognitive performance } \\
\text { and full-scale I.Q. } \\
\text { composite score } \\
\text { (WPPSI-IV), and } \\
\text { reduction in attention } \\
\text { problems (CBCL 1.5-5) }\end{array}$ \\
\hline $\begin{array}{c}\text { Metallinos- } \\
\text { Katsaras et al. } \\
\text { [58], 2004, Greece }\end{array}$ & 124 & $3-4 y$ & $\begin{array}{l}\text { Urban, high-income } \\
\text { country, children with } \\
\text { birth weight } \geq 2500 \mathrm{~g} \text {, } \\
\text { I.Q. } \geq 1 \text { s.d. below the } \\
\text { age-adjusted mean, } \\
\text { blood } \mathrm{Pb} \leq 200 \mathrm{ppb} \text {, } \\
\text { weight and head } \\
\text { circumference for the } \\
\text { age } \geq 10 \text { th percentile }\end{array}$ & $\begin{array}{l}\text { Children received } 15 \mathrm{mg} \\
\text { of iron and a } \\
\text { multivitamins } \\
\text { supplement (MV) five } \\
\text { days per week at their } \\
\text { respective day care } \\
\text { center }\end{array}$ & $\begin{array}{l}\text { Children received } \\
\text { only the } \\
\text { multivitamins } \\
\text { supplement (MV) } \\
\text { five days per } \\
\text { week at their } \\
\text { respective day } \\
\text { care center }\end{array}$ & 2 months & $\begin{array}{l}\text { Simple reaction } \\
\text { time test, CPT \& } \\
\text { O.L. tasks }\end{array}$ & $\begin{array}{l}\text { Speed of information } \\
\text { processing, speed of } \\
\text { discrimination, the } \\
\text { accuracy of } \\
\text { discrimination, and rate } \\
\text { of conceptual learning }\end{array}$ & $\begin{array}{l}\text { Iron-deficient children } \\
\text { who received iron } \\
\text { supplementation } \\
\text { showed } 14 \% \text { increase in } \\
\text { discrimination speed } \\
\text { and } 8 \% \text { improvement in } \\
\text { the accuracy domain. } \\
\text { No effects in cognitive } \\
\text { functioning were seen in } \\
\text { good iron status } \\
\text { children }\end{array}$ \\
\hline $\begin{array}{l}\text { Ogunlade et al. } \\
\text { [64], 2011, } \\
\text { South Africa }\end{array}$ & 151 & $3-6.5 y$ & $\begin{array}{c}\text { Urban, low } \\
\text { socioeconomic status, } \\
\text { children with } \\
\mathrm{Hb} \leq 12.5 \mathrm{~g} / \mathrm{dl} \text { l, all } \\
\text { children received } \\
\text { anthelmintic }\end{array}$ & $\begin{array}{l}\text { Children consumed } 35 \mathrm{~g} \\
\text { of stiff maize-meal } \\
\text { porridge with added } \\
\text { micronutrient powder }(8 \\
\text { g) containing } \\
\text { amylase-rich light } \\
\text { malted barley flour } 5 \\
\text { days per week }\end{array}$ & $\begin{array}{c}\text { Children } \\
\text { consumed } 28 \mathrm{~g} \text { of } \\
\text { soft maize-meal } \\
\text { with added } \\
\text { placebo powder } \\
(8 \mathrm{~g}) 5 \text { days per } \\
\text { week }\end{array}$ & 2.7 months & $\begin{array}{l}\text { MPI, KABC-II, } \\
\text { Atlantis \& NVI }\end{array}$ & $\begin{array}{l}\text { Learning abilities, } \\
\text { sequential and } \\
\text { simultaneous } \\
\text { processing, and } \\
\text { intellectual functioning }\end{array}$ & $\begin{array}{l}\text { Intervention children } \\
\text { showed significantly } \\
\text { higher conceptual } \\
\text { thinking abilities, higher } \\
\text { MPI and NVI scores }\end{array}$ \\
\hline $\begin{array}{l}\text { Ryan and Nelson } \\
{[59], 2008 \text {, USA }}\end{array}$ & 175 & $4 \mathrm{y}$ & $\begin{array}{l}\text { High-income country, } \\
\text { healthy children } \\
\text { consuming }<6 \text { oz of fish } \\
\text { per week, English } \\
\text { speakers, between 10th } \\
\text { and 95th percentiles for } \\
\text { weight and height, and } \\
\text { currently not taking } \\
\text { LC-PUFA supplements } \\
\text { or consuming LC-PUFA } \\
\text { fortified foods }\end{array}$ & $\begin{array}{c}\text { Children received } 400 \\
\text { mg of DHA } \\
\text { supplementation as two } \\
\text { 200-mg } \\
\text { bubblegum-flavored } \\
\text { softgel chewable }\end{array}$ & $\begin{array}{l}\text { Children received } \\
\text { capsules or } \\
\text { placebo of } \\
\text { high-oleic } \\
\text { sunflower oil } \\
\text { supplied as } 2 \text { soft } \\
\text { capsules }\end{array}$ & 4 months & PPVT \& kCPT & $\begin{array}{l}\text { Memory, attention, } \\
\text { vocabulary, processing } \\
\text { speed, response time, } \\
\text { listening skills, and } \\
\text { verbal ability }\end{array}$ & $\begin{array}{l}\text { There was no significant } \\
\text { difference between } \\
\text { groups in Leiter-R Test } \\
\text { of Sustained Attention, } \\
\text { (PPVT), Day-Night } \\
\text { Stroop Test, and (kCPT). } \\
\text { Regression analysis } \\
\text { showed a significant } \\
\text { positive association } \\
\text { between levels of DHA } \\
\text { in capillary whole blood } \\
\text { and improved listening } \\
\text { comprehension and } \\
\text { vocabulary (PPVT) }\end{array}$ \\
\hline $\begin{array}{l}\text { Black et al. [65], } \\
\text { 2021, India }\end{array}$ & 321 & $3-5 y$ & $\begin{array}{c}\text { Rural, low } \\
\text { socioeconomic status, } \\
\text { children living in a } \\
\text { district with prevalence } \\
\text { of anemia }>70 \%, 50 \% \\
\text { of children consumed } \\
<50 \% \text { of the } \\
\text { recommended intake of } \\
\text { several essential } \\
\text { micronutrients, } \\
\text { high-quality and } \\
\text { low-quality preschools } \\
\text { were included }\end{array}$ & $\begin{array}{c}\text { Children received 300g } \\
\text { of cooked food fortified } \\
\text { with } \mathrm{MNP}(13 \mathrm{mg} \text { iron, } 5 \\
\mathrm{mg} \text { zinc, } 20 \mu \mathrm{g} \\
\text { folic acid, } 150 \mu \mathrm{g} \\
\text { vitamin } \mathrm{A}, 20 \mathrm{mg} \\
\text { vitamin C, } 0.5 \mu \mathrm{g} \\
\text { vitamin B-12, and } 0.5 \mathrm{mg} \\
\text { riboflavin }\end{array}$ & $\begin{array}{l}\text { Children received } \\
300 \mathrm{~g} \text { of cooked } \\
\text { food containing } \\
0.5 \mathrm{mg} \text { riboflavin } \\
\text { (no effects on } \\
\text { outcome } \\
\text { measures) }\end{array}$ & 8 months & MSEL \& BSID-III & $\begin{array}{l}\text { Fine motor skills, gross } \\
\text { motor skills, visual } \\
\text { reception, receptive } \\
\text { language, expressive } \\
\text { language, and } \\
\text { social-emotional } \\
\text { behaviors }\end{array}$ & $\begin{array}{l}\text { For children attending } \\
\text { low-quality preschools, } \\
\text { MNP fortification } \\
\text { improved expressive } \\
\text { language and } \\
\text { marginally improved } \\
\text { inhibitory control and } \\
\text { social-emotional } \\
\text { development in } \\
\text { comparison to children } \\
\text { attending control } \\
\text { low-quality preschools. } \\
\text { MNP fortification did } \\
\text { not impact any area of } \\
\text { cognitive development } \\
\text { in children attending } \\
\text { high-quality preschools }\end{array}$ \\
\hline $\begin{array}{c}\text { Kvestad et al. [60], } \\
\text { 2018, } \\
\text { Norway }\end{array}$ & 232 & $4-6 y$ & $\begin{array}{l}\text { High-income country, } \\
\text { healthy children with no } \\
\text { food allergies }\end{array}$ & $\begin{array}{l}\text { Children received lunch } \\
\text { meals containing } 50-80 \\
\text { g of fatty fish } \\
\text { (herring/mackerel) } \\
\text { three times per week }\end{array}$ & $\begin{array}{l}\text { Children received } \\
\text { lunch meals } \\
\text { containing 50-80 } \\
\text { g of meat } \\
\text { (chicken/lamb/beef) } \\
\text { three times per } \\
\text { week }\end{array}$ & 4 months & WPPSI-III & $\begin{array}{l}\text { Information, vocabulary, } \\
\text { block design, word and } \\
\text { matrix reasoning, } \\
\text { picture concepts, coding, } \\
\text { and symbol search }\end{array}$ & $\begin{array}{l}\text { No significant difference } \\
\text { between groups }\end{array}$ \\
\hline
\end{tabular}


Table 2. Cont.

\begin{tabular}{|c|c|c|c|c|c|c|c|c|c|}
\hline $\begin{array}{l}\text { Reference, Year, } \\
\text { Country }\end{array}$ & $\begin{array}{c}\text { Sample } \\
\text { Size }\end{array}$ & Age & $\begin{array}{l}\text { Subject Characteristics } \\
\text { at Baseline }\end{array}$ & Intervention Group/s & Control Group/s & Duration & Cognitive Tests & $\begin{array}{c}\text { Cognitive Domain } \\
\text { Assessed }\end{array}$ & $\begin{array}{l}\text { Major Cognitive } \\
\text { Outcomes }\end{array}$ \\
\hline $\begin{array}{c}\text { Roberts et al. [66], } \\
2020, \\
\text { Guinea-Bissau }\end{array}$ & 1059 & $1.3-7 \mathrm{y}$ & $\begin{array}{c}\text { Rural, low } \\
\text { socioeconomic status, } \\
\text { children living in one of } \\
\text { the } 10 \text { rural villages in } \\
\text { the Oio and Cacheu } \\
\text { regions of } \\
\text { Guinea-Bissau, children } \\
\text { with severe acute } \\
\text { malnutrition or relevant } \\
\text { food allergies were } \\
\text { excluded from the study }\end{array}$ & $\begin{array}{l}\text { One group of children } \\
\text { received NEWSUP } \\
(\approx 310 \text { kcal) for breakfast } \\
\text { as a raw paste } \\
\text { containing } 98 \% \text { of } \\
\text { recommended daily } \\
\text { micronutrients for } \\
\text { children under } 4 \text { y, the } \\
\text { second group received } \\
\text { FBF }(\approx 310 \text { kcal) for } \\
\text { breakfast served as a } \\
\text { corn soy blend with } \\
\text { cooked porridge, } \\
\text { fortified oil, sugar, and } \\
\text { salt containing an } \\
\text { average of } 16 \% \\
\text { recommended daily } \\
\text { micronutrients }\end{array}$ & $\begin{array}{c}\text { Children received } \\
\text { white rice cooked } \\
\text { in water, soybean } \\
\text { oil, and salt }(\approx 310 \\
\text { kcal) containing } \\
\text { an average of } 1 \% \\
\text { of recommended } \\
\text { daily } \\
\text { micronutrients }\end{array}$ & 5.7 months & $\begin{array}{l}\text { Working Memory } \\
\text { Task Test }\end{array}$ & Working memory & $\begin{array}{c}\text { Intervention children } \\
\text { younger than } 4 \mathrm{y} \\
\text { receiving NEWSUP } \\
\text { displayed increased } \\
\text { working memory } \\
\text { compared to control } \\
\text { children }\end{array}$ \\
\hline
\end{tabular}

Randomized Controlled Trials (RCTs); Wechsler Pre-school and Primary Scale of Intelligence (WPPSI); Kaufman Assessment Battery for Children (KABC); Intelligence Quotient (I.Q.); Urine Iodine Concentration (UIC); 9-Hole Peg Test (9-HPT); Full Scale I.Q. (FIQ); Performance Intelligence Quotient (PIQ); Integrated Child Development Services (ICDS); Mullen Scales of Early Learning (MSEL); Standard Deviation (SD); Eicosapentaenoic acid (EPA); Docosahexaenoic acid (DHA); Calories (kcal); Child Behavior Checklist (CBCL); Parenting Interactions with Children: Checklist of Observations Linked to Outcomes (PICCOLO); Multivitamin (MV); Cognitive Performance Test (CPT); Oddity Learning (O.L.); Multidimensional Prognostic; Index (MPI); Nonverbal Index (NVI); Longchain polyunsaturated fatty acids (LC-PUFAs); Polyunsaturated Fatty Acids (PUFAs); Peabody Picture Vocabulary Test (PPVT); Conners Kiddie; Continuous Performance Test (kCPT); Bayley Scales of Infant Development (BSID); Multiple-micronutrient (MMN); Point-of-use multiple micronutrient powder (MNP). y: years old.

\subsection{Study Quality}

Six studies had an overall low risk of bias $[55,56,59,60,65,66]$ and six had a moderate risk of bias [57,58,61-64] as indicated in Table 3. Five studies did not record methods of handling withdrawal $[57,58,61-63]$ and two studies $[57,64]$ reported moderate differences between study groups at baseline.

Table 3. Quality Criteria Checklist (QCC; risk of bias) assessment of each study included in the review [55-66].

\begin{tabular}{|c|c|c|c|c|c|c|c|c|c|c|c|c|}
\hline First Author, Year of Publication (Reference) & $\begin{array}{l}\text { Rauh- } \\
\text { Pfeiffer, } \\
2014 \\
{[55]}\end{array}$ & $\begin{array}{l}\text { Demmelmair, } \\
2019[56]\end{array}$ & $\begin{array}{l}\text { Ogunlade, } \\
2011[64]\end{array}$ & $\begin{array}{l}\text { Choudhury, } \\
2021[62]\end{array}$ & $\begin{array}{c}\text { Black, } \\
2021[65]\end{array}$ & $\begin{array}{l}\text { Kvestad, } \\
2018[60]\end{array}$ & $\begin{array}{l}\text { Roberts, } \\
2020[66]\end{array}$ & $\begin{array}{l}\text { Katsaras, } \\
2004[58]\end{array}$ & $\begin{array}{l}\text { Aboud, } \\
2017[61]\end{array}$ & $\begin{array}{l}\text { Øyen, } \\
2018[57]\end{array}$ & $\begin{array}{l}\text { Schneider, } \\
2018[63]\end{array}$ & $\begin{array}{l}\text { Ryan, } \\
2008[59]\end{array}$ \\
\hline \multicolumn{13}{|l|}{ Primary Research QCC } \\
\hline 1. Was the research question clearly stated? & $\mathrm{Y}$ & $\mathrm{Y}$ & $\mathrm{Y}$ & $\mathrm{Y}$ & $\mathrm{Y}$ & $\mathrm{Y}$ & $\mathrm{Y}$ & $\mathrm{Y}$ & $\mathrm{Y}$ & $\mathrm{Y}$ & $\mathrm{Y}$ & Y \\
\hline $\begin{array}{l}\text { 2. Was the selection of study subjects/patients free } \\
\text { from bias? }\end{array}$ & $\mathrm{Y}$ & $\mathrm{Y}$ & $\mathrm{Y}$ & Y & $\mathrm{Y}$ & $\mathrm{Y}$ & $\mathrm{Y}$ & $\mathrm{Y}$ & Y & Y & $\mathrm{Y}$ & Y \\
\hline 3. Were study groups comparable? & $\mathrm{Y}$ & $\mathrm{Y}$ & $\mathrm{N}$ & $\mathrm{Y}$ & $\mathrm{Y}$ & $\mathrm{Y}$ & $\mathrm{Y}$ & $\mathrm{Y}$ & $\mathrm{N}$ & $\mathrm{Y}$ & $\mathrm{Y}$ & $\mathrm{Y}$ \\
\hline 4. Was method of handling withdrawals described? & $\mathrm{Y}$ & $\mathrm{Y}$ & $\mathrm{Y}$ & $\mathrm{N}$ & $\mathrm{Y}$ & $\mathrm{Y}$ & $\mathrm{Y}$ & $\mathrm{N}$ & $\mathrm{N}$ & $\mathrm{N}$ & $\mathrm{N}$ & $\mathrm{Y}$ \\
\hline 5. Was blinding used to prevent introduction of bias? & $\mathrm{Y}$ & $\mathrm{N}$ & $\mathrm{Y}$ & $\mathrm{Y}$ & $Y$ & $\mathrm{Y}$ & $\mathrm{N}$ & $Y$ & $\mathrm{~N}$ & $Y$ & $\mathrm{~N}$ & $Y$ \\
\hline $\begin{array}{l}\text { 6. Were intervention/exposure factor or procedure and } \\
\text { any comparison(s) described in detail? }\end{array}$ & $\mathrm{Y}$ & $\mathrm{N}$ & $\mathrm{Y}$ & $\mathrm{Y}$ & $Y$ & $Y$ & $\mathrm{Y}$ & $Y$ & $Y$ & $Y$ & $Y$ & $\mathrm{Y}$ \\
\hline $\begin{array}{l}\text { 7. Were outcomes clearly defined and the } \\
\text { measurements valid and reliable? }\end{array}$ & $\mathrm{Y}$ & $Y$ & $\mathrm{Y}$ & $Y$ & $\mathrm{Y}$ & $Y$ & $\mathrm{Y}$ & $\mathrm{Y}$ & $Y$ & $\mathrm{Y}$ & $\mathrm{Y}$ & $\mathrm{N}$ \\
\hline $\begin{array}{l}\text { 8. Was the statistical analysis appropriate for the study } \\
\text { design and type of outcome indicators? }\end{array}$ & $Y$ & $\mathrm{Y}$ & $\mathrm{Y}$ & $\mathrm{Y}$ & $\mathrm{Y}$ & $\mathrm{N}$ & $\mathrm{Y}$ & $\mathrm{Y}$ & $\mathrm{Y}$ & $\mathrm{Y}$ & $\mathrm{Y}$ & $\mathrm{Y}$ \\
\hline $\begin{array}{l}\text { 9. Were conclusions supported by results with biases } \\
\text { and limitations taken into consideration? }\end{array}$ & $\mathrm{N}$ & $Y$ & $\mathrm{Y}$ & $\mathrm{Y}$ & $\mathrm{Y}$ & $\mathrm{Y}$ & $\mathrm{Y}$ & $\mathrm{Y}$ & $\mathrm{Y}$ & $\mathrm{Y}$ & $\mathrm{Y}$ & $\mathrm{Y}$ \\
\hline $\begin{array}{l}\text { 10. Is bias due to study's funding or sponsorship } \\
\text { unlikely? }\end{array}$ & $\mathrm{Y}$ & $Y$ & $\mathrm{Y}$ & $\mathrm{Y}$ & $Y$ & $Y$ & $\mathrm{Y}$ & ? & $Y$ & $Y$ & $\mathrm{~N}$ & $Y$ \\
\hline OVERALL QUALITY & $(+)$ & $(+)$ & (Ø) & (Ø) & $(+)$ & $(+)$ & $(+)$ & (Ø) & (Ø) & (Ø) & (Ø) & $(+)$ \\
\hline
\end{tabular}

Plus/positive (+); neutral (Ø).

\subsection{Study Participants}

The study populations were comprised mostly of preschool-age healthy children. However, children with insufficient folate levels, children at risk of undernutrition and micronutrient deficiencies, children with anemia, and children receiving anthelmintic medication were also included. 


\subsection{Nutritional Interventions}

Supplement-based interventions were adopted in five out of twelve studies. These interventions included guava supplementation [62], DHA tablets [59], iron supplementation [58], B vitamin sachets [55], and iodized salt [61]. Multiple-micronutrient (MMN) food fortification interventions were implemented in three studies. For MMN interventions, the fortification was added to maize-porridge [64], rice, or wheat [65], and provided as a raw paste [66]. A total of three studies included food-based nutritional interventions in which subjects consumed fatty fish with daily meals $[56,57,60]$. Finally, one study conducted a dietary intervention in the form of a fortified milk powder [63], combined with cognitive stimulation.

\subsection{Cognitive Tests}

A diverse range of standardized cognitive tests were used to assess outcomes in multiple cognitive domains, including learning abilities, verbal reasoning, intellectual functioning, information processing speed, vocabulary, word reasoning, speed and accuracy of discrimination, fine and gross motor skills, coding, symbol search, and working memory. Half of the studies administered the Wechsler Pre-school and Primary Scale of Intelligence (WPPSI) test $[55-57,60,61,63]$, two experimental studies administered the 9-Hole Peg Test (9-HPT) [56,57], and two studies administered the Mullen Scale of Early Learning (MSEL) [59,62]. Table 2 includes a detailed description of cognitive tests used in each trial, cognitive domains assessed, and effects on cognitive outcomes of preschool-aged children.

\subsection{Major Cognitive Outcomes}

\subsubsection{Single Nutrient Supplementation}

Five RCTs measured the effect of supplement-based interventions on children's cognition. Three trials failed to find a significant impact on cognition: the B vitamin [55], iodized salt [59], and guava [62] supplementation interventions. Guava is a fruit high in several vitamins and minerals and is also rich in lycopene, a carotenoid phytonutrient known for its antioxidant effect [67]. Guava was used as an intervention due to its high vitamin C content and its effects in facilitating nonheme iron absorption [68]. Although Guava supplementation yielded significant improvements in the iron status of children, no significant effects were seen in cognitive function [68]. Out of the two trials that found significant results, one of them was the iron intervention [58], which found that for children with iron deficiency anemia, iron supplementation increased accuracy and the speed of discrimination on the continuous performance task; however, for children with adequate iron status at baseline, iron supplementation did not affect performance on the continuous processing task. The DHA supplementation intervention [59] did not find any significant differences in cognitive function scores between the intervention and placebo groups; however, higher blood DHA levels were significantly associated with higher scores on the Peabody Picture Vocabulary Test, which measures listening comprehension and vocabulary.

\subsubsection{Multiple-Micronutrient Supplementation}

Three RCTs measured the impact of multiple-micronutrient food fortification on children's cognitive development. In the 11-week intervention consisting of eight grams of a point of use multiple micronutrient powder added to maize-meal porridge at breakfast for children 36-79 months of age [64], children in the intervention group significantly increased their scores on the simultaneous scale and the non-verbal index of the Kaufman Assessment Battery for Children compared to the control group. Conversely, in another longer trial where the children were somewhat younger [65], the cognitive benefits of a point of use multiple micronutrient powder were dependent upon whether the child was attending a low-quality or a high-quality preschool. Here, the quality of preschools was assessed by combining two validated scales, the Early Childhood Environment Rating Scale-Revised and the Home Observation for the Measurement of the Environment (HOME) adjusted for teachers rather than parents. Preschool quality was determined by two psychologists who 
independently assessed preschools as low or high-quality based on playing space, learning opportunities available to children, organization of environment, teacher-child interaction levels, and involvement levels of preschool-age children to activities and practices offered. Children from low-quality preschools who received the micronutrient powder fortification displayed improvements in expressive language and some, but to a lesser degree, improvement in inhibitory control and social-emotional development, whereas children attending high-quality pre-schools showed no improvements in cognitive outcomes. In the New Multicomponent Supplementary Food (NEWSUP) study [66], children 15-48 months of age were given a unique supplementary food that contained not just multiple micronutrients, but also plant polyphenols, omega-3 fatty acids, and protein for 23 weeks. Among the children younger than four years of age, the intervention group significantly increased working memory compared to the control group. However, among children 4-7 years of age who were administered the same intervention, no improvements in working memory were seen.

\subsubsection{Food-Based Interventions}

Three RCTs evaluated the impact of food-based interventions on children's cognition. All trials involved the provision of meals containing fatty fish. Among children aged 4-6 years enrolled in kindergartens in Germany, children who consumed Atlantic salmon three times per week for 16 weeks saw modest improvements in two indicators of nonverbal fluid intelligence that were greater than the improvements seen in the control group who received beef in place of salmon [56]. The salmon intervention did not improve total IQ scores, but the slight improvement in raw scores over the beef group in two subcomponents suggests that provision of fatty fish may offer benefits to certain specific aspects of cognitive function. In the FINS-KIDS trial, a similarly designed study that used the same measurements of cognitive function as the aforementioned study (the WPPSI-III and the 9-HPT), provision of herring and mackerel to 4-6-year-old children in Norway increased total raw scores on the WPPSI-III when analyses were adjusted for dietary compliance [57]. When looking at individual sub-tests of the WPPSI-III, the fatty fish group showed improved performance on three of the sub-tests: the symbol search test, which is one of the sub-tests that showed improved scores in the Atlantic salmon trial, and the vocabulary and block design sub-tests. The FINS-KIDS trial also examined whether there was any association between changes in total hair mercury concentrations after the fatty fish intervention and cognition [60] and found that while the herring/mackerel intervention did increase mercury levels, the values remained below a level of concern and were not associated with cognitive function.

\subsubsection{Effects of Nutritional Intervention Combined with Psychosocial Stimulation}

One RCT [63] measured the impact of dietary intervention combined with psychosocial stimulation on children's cognitive development. This trial was conducted in Indonesia and included children 3-5 years of age who had a below-average level of stimulation in the home. Children receiving a fortified milk powder supplement who performed psychosocial stimulation activities three times per week [63] demonstrated a larger increase in the full-scale IQ composite score component of the WPPSI-IV compared to the control group; however, none of the IQ sub scores were different between the intervention and control group. Parents of children in the experimental group additionally reported a larger reduction in attention problems compared to that reported for children in the control group.

\section{Discussion}

This review suggests nutritional interventions significantly improve cognitive outcomes of undernourished preschool-age children. Trials conducted in LMICs demonstrated that nutrient-deprived children who received dietary interventions consistently showed improvements in cognition. However, caution should be taken when interpreting findings due to the clinical heterogeneity of eligible studies. Metallinos-Katsaras et al. [58] suggests 
preschool children with anemia who receive iron supplementation can process information faster while making fewer mistakes. These findings are consistent with another systematic review [69]. According to Roberts et al. [66], nutrient-deprived children in the intervention group of the NEWSUP study showed improved working memory. Ogunlade et al. [64] stated that undernourished children who consumed point-of-use fortification displayed superior information analysis and problem-solving skills and improved mental processing abilities. Black et al. [65] noted that children attending low-quality preschools who received a multiple micronutrient powder with their meals improved expressive language. Considering the detrimental effects of nutrient deficiencies on children's cognitive, social, and emotional skills, multiple micronutrient supplementation is a promising intervention to re-establish nutrient balance and to increase the developmental potential of undernourished preschool-age children. Although the first 1000 days of life are well-established as the most critical period for brain development and growth in a child's life, this review suggests the second 1000 days to also be a critical period in cognitive development. Thus, preschool-age children at risk for nutrient deficiencies who receive dietary intervention can further develop their cognitive abilities. Although promising outcomes can be achieved from nutrient supplementation interventions, operational, monetary, and sustainability challenges might hinder supplement-based trial design and implementation. Additionally, multiple-micronutrient fortification intervention trials discussed in this review yielded positive effects on cognition in the short-term, and little research exists regarding the long-term efficacy of such interventions.

This review indicates well-nourished children benefit from increased fish consumption. Demmelmair et al. [56] found that healthy children in the intervention group who consumed fish increased their full-scale IQ scores from baseline, and Øyen et al. [57] found that children displayed improved processing speed abilities with fish consumption. The benefits of increased fish consumption on the cognitive development of preschool-age children identified in this review are consistent with the findings of another review [70]. On the other hand, three studies that implemented single nutrient supplement-based interventions, B vitamin sachets [55], iodized salt [61], and guava [62] showed no significant benefits associated with supplementation on cognitive outcomes of preschool-aged children. Of the studies included in this review, only 25\% measured the effect of real food interventions on children's cognition, and of these, all were conducted in high-income countries and solely interested in the impact of fatty fish and DHA on children's cognition.

To our knowledge, there were no trials designed to explore the benefits of increased vegetable and fruit consumption on the cognitive performance of preschool-age children. Furthermore, no study measured the effect of improved dietary diversity on preschoolers' cognitive development. Dietary diversity is adequate when a child's diet contains five or more of the eight recommended food groups, including breast milk, grains, roots and tubers, legumes and nuts, dairy products (milk, yogurt, cheese), flesh foods (meat, fish, poultry, liver, or other organs), eggs, vitamin A-rich fruits and vegetables, and other fruits and vegetables $[53,71]$. Dietary diversity is the preferred approach to improving the nutrition of a population, as it is the most sustainable and desirable approach [71]. Therefore, future trials must include interventions intended to explore the effects of increased dietary diversity on the cognitive development of young children.

Additionally, further evidence is needed to determine whether nutritional interventions combined with psychosocial stimulation result in superior cognitive outcomes. For example, Schneider et al. [63] suggested that nutritional supplementation with low-level cognitive stimulation yields improved cognitive functioning in preschool-age children. However, considering as many as 200 million children in the world lack access to sufficient nutrition and adequate cognitive stimulation [5], trials that combine psychosocial stimulations and nutrient interventions on the cognitive development of preschool-age children are needed to provide more definite evidence on the impact of a combined intervention approach on cognitive outcomes. 
Further research is also required to investigate the long-term effects of multiple micronutrient supplementation in cognitive outcomes of nutrient-deprived children, as shortterm benefits have been consistently demonstrated. In addition, more research is needed to explore the effects of nutritional interventions combined with psychosocial stimulation on cognitive outcomes of preschool-aged children. This evidence is essential, because cognitive development reached in preschool years often determines school readiness and predicts later life achievements [5].

\section{Conclusions}

In conclusion, the findings of this review show that nutritional interventions have a positive effect on the cognitive development of undernourished preschool-age children. Nutrient-deficient children who receive micronutrient supplementation consistently display significant advances in cognitive outcomes. Furthermore, nourished children who increase fish consumption display improvements in cognitive abilities. This review highlights the importance of adequate nutrient intake during the second 1000 days of a child's life, and the crucial role sufficient nutrition plays in cognitive development.

Author Contributions: Conceptualization, T.T.-P., M.R., and N.R.; methodology, M.R.; formal analysis, M.R.; investigation, M.R., A.R., and C.W.; data curation, M.R.; writing-original draft preparation, M.R.; writing—review and editing, M.R., T.T.-P., N.R., and G.R.M.; supervision, T.T.-P. and N.R. All authors have read and agreed to the published version of the manuscript.

Funding: This research was funded by the United States Department of Agriculture-Foreign Agricultural Services. FX21TA-10960R003.

Institutional Review Board Statement: Not applicable.

Informed Consent Statement: Not applicable.

Acknowledgments: This study was supported by the Mississippi State University Department of Food Science, Nutrition, and Health Promotion.

Conflicts of Interest: The authors declare no conflict of interest.

\section{Appendix A}

Table A1. PICO framework: keywords.

\begin{tabular}{|c|c|c|c|}
\hline Population & Intervention & Comparison & Outcome \\
\hline $\begin{array}{l}\text { Preschool children } \\
\text { ( } 2-6 \text { years of age) }\end{array}$ & $\begin{array}{l}\text { Multiple-micronutrient } \\
\text { (MMN) food fortification, } \\
\text { supplement-based, or } \\
\text { food-based nutritional } \\
\text { interventions }\end{array}$ & Placebo or control group & $\begin{array}{c}\text { Cognitive outcomes } \\
\text { using cognitive } \\
\text { assessment tests }\end{array}$ \\
\hline
\end{tabular}

Table A2. Search Strategy used for each electronic database.

\begin{tabular}{cc}
\hline Database & Searched Terms \\
\hline & (nutrient OR nutrition OR micronutrient OR macronutrient OR diet OR \\
dietary OR "food intake" OR "meal diversity") AND ("child \\
development" OR cognition OR focus OR brain OR attentiveness OR \\
attention OR memory OR verbal OR vocabulary OR learning OR literacy \\
OR neuro* OR problem-solving OR reasoning OR "school performance" \\
OR "school achievement" OR "academic achievement" OR "educational \\
measurement" OR "academic success" OR "academic performance") \\
AND (randomized controlled trial [pt] OR controlled clinical trial [pt] OR \\
randomized [tiab] OR placebo [tiab] OR clinical trials OR randomly [tiab] \\
OR trial [ti]) AND (child OR children OR preschool OR "pre-school")
\end{tabular}


Table A2. Cont.

\begin{tabular}{cc}
\hline Database & Searched Terms \\
\hline CENTRAL & (nutrient* OR nutrition* OR micronutrient* OR macronutrient* OR diet* \\
OR "food intake" OR "meal diversity") AND ("child development" OR \\
cognition OR focus OR brain OR attentiveness OR attention OR memory \\
OR verbal OR vocabulary OR learning OR literacy OR neuro* OR \\
problem-solving OR reasoning OR "school performance" OR "school \\
achievement" OR "academic achievement" OR "educational \\
measurement") AND (child OR children OR preschool OR "pre-school")
\end{tabular}

\section{References}

1. De Onis, M.B.M. Quantifying the Health Impact at National and Local Levels. Available online: https://www.who.int/ quantifying_ehimpacts/publications/MalnutritionEBD12.pdf (accessed on 31 August 2021).

2. World Health Organization (WHO). Malnutrition Fact Sheets. Available online: https://www.who.int/news-room/fact-sheets/ detail/malnutrition (accessed on 20 July 2021).

3. De Onís, M.; Monteiro, C.; Akré, J.; Glugston, G. The Worldwide Magnitude of Protein-Energy Malnutrition: An Overview from the WHO Global Database on Child Growth. Bull. World Health Organ. 1993, 71, 703-712. [PubMed]

4. World Health Organization. UNICEF/WHO/The World Bank Group Joint Child Malnutrition Estimates: Levels and Trends in Child Malnutrition: Key Findings of the 2021 Edition. Available online: https://www.who.int/publications/i/item/9789240025 257 (accessed on 31 July 2021).

5. Grantham-McGregor, S. A Review of Studies of the Effect of Severe Malnutrition on Mental Development. J. Nutr. 1995, 125 (Suppl. S8), 2233S-2238S. [CrossRef] [PubMed]

6. Wachs, T.D. Relation of Mild-to-Moderate Malnutrition to Human Development: Correlational Studies. J. Nutr. 1995, 125 (Suppl. S8), 2245S-2254S. [CrossRef] [PubMed]

7. Cusick, S.E.; Georgieff, M.K. The Role of Nutrition in Brain Development: The Golden Opportunity of the "First 1000 Days". J. Pediatr. 2016, 175, 16-21. [CrossRef] [PubMed]

8. $\quad$ Black, R.E.; Victora, C.G.; Walker, S.P.; Bhutta, Z.A.; Christian, P.; de Onis, M.; Ezzati, M.; Grantham-McGregor, S.; Katz, J.; Martorell, R.; et al. Maternal and Child Nutrition Study Group. Maternal and Child Undernutrition and Overweight in Low-Income and Middle-Income Countries. Lancet 2013, 382, 427-451. [CrossRef]

9. Sigman, M.; McDonald, M.A.; Neumann, C.; Bwibo, N. Prediction of Cognitive Competence in Kenyan Children from Toddler Nutrition, Family Characteristics and Abilities. J. Child. Psychol. Psychiatry 1991, 32, 307-320. [CrossRef] [PubMed]

10. Martins, V.J.B.; Toledo Florêncio, T.M.M.; Grillo, L.P.; do Carmo P Franco, M.; Martins, P.A.; Clemente, A.P.G.; Santos, C.D.L.; de Fatima A Vieira, M.; Sawaya, A.L. Long-Lasting Effects of Undernutrition. Int. J. Environ. Res. Public Health 2011, 8, 1817-1846. [CrossRef]

11. Mehta, N.M.; Corkins, M.R.; Lyman, B.; Malone, A.; Goday, P.S.; Carney, L.N.; Monczka, J.L.; Plogsted, S.W.; Schwenk, W.F.; American Society for Parenteral and Enteral Nutrition Board of Directors. Defining Pediatric Malnutrition: A Paradigm Shift toward Etiology-Related Definitions: A Paradigm Shift toward Etiology-Related Definitions. JPEN J. Parenter. Enter. Nutr. 2013, 37, 460-481. [CrossRef]

12. Olsen, E.M. Failure to Thrive: Still a Problem of Definition. Clin. Pediatr. 2006, 45, 1-6. [CrossRef]

13. Homan, G.J. Failure to Thrive: A Practical Guide. Am. Fam. Physician 2016, 94, 295-299.

14. Bourre, J.M. Effects of Nutrients (in Food) on the Structure and Function of the Nervous System: Update on Dietary Requirements for Brain. Part 1: Micronutrients. J. Nutr. Health Aging 2006, 10, 377-385. [PubMed]

15. Monk, C.; Georgieff, M.K.; Osterholm, E.A. Research Review: Maternal Prenatal Distress and Poor Nutrition-Mutually Influencing Risk Factors Affecting Infant Neurocognitive Development: Maternal Prenatal Distress and Poor Nutrition. J. Child. Psychol. Psychiatry 2013, 54, 115-130. [CrossRef] [PubMed]

16. Pollitt, E.; Gorman, K.S.; Engle, P.L.; Rivera, J.A.; Martorell, R. Nutrition in Early Life and the Fulfillment of Intellectual Potential. J. Nutr. 1995, 125 (Suppl. S4), 1111S-1118S. [PubMed]

17. Muñoz, P.; Humeres, A. Iron Deficiency on Neuronal Function. Biometals 2012, 25, 825-835. [CrossRef]

18. Todorich, B.; Pasquini, J.M.; Garcia, C.I.; Paez, P.M.; Connor, J.R. Oligodendrocytes and Myelination: The Role of Iron. Glia 2009, 57, 467-478. [CrossRef]

19. Youdim, M.B.; Yehuda, S. The Neurochemical Basis of Cognitive Deficits Induced by Brain Iron Deficiency: Involvement of Dopamine-Opiate System. Cell. Mol. Biol. 2000, 46, 491-500.

20. Abbaspour, N.; Hurrell, R.; Kelishadi, R. Review on Iron and Its Importance for Human Health. J. Res. Med. Sci. 2014, 19, 164-174. 
21. McCann, S.; Perapoch Amadó, M.; Moore, S.E. The Role of Iron in Brain Development: A Systematic Review. Nutrients 2020, 12, 2001. [CrossRef]

22. Black, M.M. Zinc Deficiency and Child Development. Am. J. Clin. Nutr. 1998, 68 (Suppl. S2), 464S-469S. [CrossRef]

23. Golub, M.S.; Keen, C.L.; Gershwin, M.E.; Hendrickx, A.G. Developmental Zinc Deficiency and Behavior. J. Nutr. 1995, 125 (Suppl. S8), 2263S-2271S. [CrossRef]

24. Prasad, A.S. Impact of the Discovery of Human Zinc Deficiency on Health. J. Am. Coll. Nutr. 2009, 28, 257-265. [CrossRef] [PubMed]

25. Prasad, A.S. Discovery of Human Zinc Deficiency and Studies in an Experimental Human Model. Am. J. Clin. Nutr. 1991, 53, 403-412. [CrossRef] [PubMed]

26. Strain, J.J.; McSorley, E.M.; van Wijngaarden, E.; Kobrosly, R.W.; Bonham, M.P.; Mulhern, M.S.; McAfee, A.J.; Davidson, P.W.; Shamlaye, C.F.; Henderson, J.; et al. Choline Status and Neurodevelopmental Outcomes at 5 Years of Age in the Seychelles Child Development Nutrition Study. Br. J. Nutr. 2013, 110, 330-336. [CrossRef] [PubMed]

27. Gámiz, F.; Gallo, M. A Systematic Review of the Dietary Choline Impact on Cognition from a Psychobiological Approach: Insights from Animal Studies. Nutrients 2021, 13, 1966. [CrossRef] [PubMed]

28. Albright, C.D.; Friedrich, C.B.; Brown, E.C.; Mar, M.H.; Zeisel, S.H. Maternal Dietary Choline Availability Alters Mitosis, Apoptosis and the Localization of TOAD-64 Protein in the Developing Fetal Rat Septum. Brain Res. Dev. Brain Res. 1999, 115, 123-129. [CrossRef]

29. Albright, C.D.; Tsai, A.Y.; Friedrich, C.B.; Mar, M.-H.; Zeisel, S.H. Choline Availability Alters Embryonic Development of the Hippocampus and Septum in the Rat. Brain Res. Dev. Brain Res. 1999, 113, 13-20. [CrossRef]

30. Kapil, U. Health Consequences of Iodine Deficiency. Sultan Qaboos Univ. Med. J. 2007, 7, 267-272.

31. Mahan, L.K.; Raymond, J.L. Krause's Food \& The Nutrition Care Process, 14th ed.; Elsevier Inc.: St. Louis, MO, USA, 2017; pp. 257-260.

32. Crider, K.S.; Yang, T.P.; Berry, R.J.; Bailey, L.B. Folate and DNA Methylation: A Review of Molecular Mechanisms and the Evidence for Folate's Role. Adv. Nutr. 2012, 3, 21-38. [CrossRef]

33. De-Regil, L.M.; Fernández-Gaxiola, A.C.; Dowswell, T.; Peña-Rosas, J.P. Effects and Safety of Periconceptional Folate Supplementation for Preventing Birth Defects. Cochrane Database Syst. Rev. 2010, CD007950. [CrossRef]

34. Dror, D.K.; Allen, L.H. Effect of Vitamin B12 Deficiency on Neurodevelopment in Infants: Current Knowledge and Possible Mechanisms. Nutr. Rev. 2008, 66, 250-255. [CrossRef]

35. Winje, B.A.; Kvestad, I.; Krishnamachari, S.; Manji, K.; Taneja, S.; Bellinger, D.C.; Bhandari, N.; Bisht, S.; Darling, A.M.; Duggan, C.P.; et al. Does Early Vitamin B12 Supplementation Improve Neurodevelopment and Cognitive Function in Childhood and into School Age: A Study Protocol for Extended Follow-Ups from Randomised Controlled Trials in India and Tanzania. BMJ Open 2018, 8, e018962. [CrossRef] [PubMed]

36. Weiser, M.J.; Butt, C.M.; Mohajeri, M.H. Docosahexaenoic Acid and Cognition throughout the Lifespan. Nutrients 2016, 8, 99. [CrossRef] [PubMed]

37. Clandinin, M.T.; Van Aerde, J.E.; Merkel, K.L.; Harris, C.L.; Springer, M.A.; Hansen, J.W.; Diersen-Schade, D.A. Growth and Development of Preterm Infants Fed Infant Formulas Containing Docosahexaenoic Acid and Arachidonic Acid. J. Pediatr. 2005, 146, 461-468. [CrossRef] [PubMed]

38. Bourre, J.M. Roles of Unsaturated Fatty Acids (Especially Omega-3 Fatty Acids) in the Brain at Various Ages and during Ageing. J. Nutr. Health Aging 2004, 8, 163-174. [PubMed]

39. FHI Solutions LLC. Why 1000 Days. Available online: https:/ / thousanddays.org/why-1000-days/ (accessed on 31 August 2021).

40. Lenroot, R.K.; Giedd, J.N. Brain Development in Children and Adolescents: Insights from Anatomical Magnetic Resonance Imaging. Neurosci. Biobehav. Rev. 2006, 30, 718-729. [CrossRef]

41. Bryan, J.; Osendarp, S.; Hughes, D.; Calvaresi, E.; Baghurst, K.; van Klinken, J.-W. Nutrients for Cognitive Development in School-Aged Children. Nutr. Rev. 2004, 62, 295-306. [CrossRef]

42. Petry, N.; Olofin, I.; Boy, E.; Donahue Angel, M.; Rohner, F. The Effect of Low Dose Iron and Zinc Intake on Child Micronutrient Status and Development during the First 1000 Days of Life: A Systematic Review and Meta-Analysis. Nutrients 2016, 8, 773. [CrossRef]

43. Derbyshire, E.; Obeid, R. Choline, Neurological Development and Brain Function: A Systematic Review Focusing on the First 1000 Days. Nutrients 2020, 12, 1731. [CrossRef]

44. Brown, T.T.; Jernigan, T.L. Brain Development during the Preschool Years. Neuropsychol. Rev. 2012, 22, 313-333. [CrossRef]

45. Welsh, J.A.; Nix, R.L.; Blair, C.; Bierman, K.L.; Nelson, K.E. The Development of Cognitive Skills and Gains in Academic School Readiness for Children from Low-Income Families. J. Educ. Psychol. 2010, 102, 43-53. [CrossRef]

46. Grantham-McGregor, S.; Cheung, Y.B.; Cueto, S.; Glewwe, P.; Richter, L.; Strupp, B. Developmental Potential in the First 5 Years for Children in Developing Countries. Lancet 2007, 369, 60-70. [CrossRef]

47. Stith, A.Y.; Gorman, K.S.; Choudhury, N. The Effects of Psychosocial Risk and Gender on School Attainment in Guatemala. Appl. Psychol. 2003, 52, 614-629. [CrossRef]

48. Gupta, R.P.-S.; de Wit, M.L.; McKeown, D. The Impact of Poverty on the Current and Future Health Status of Children. Paediatr. Child. Health 2007, 12, 667-672. [CrossRef] [PubMed] 
49. Kern, M.L.; Friedman, H.S. Early Educational Milestones as Predictors of Lifelong Academic Achievement, Midlife Adjustment, and Longevity. J. Appl. Dev. Psychol. 2008, 30, 419-430. [CrossRef] [PubMed]

50. Page, M.J.; McKenzie, J.E.; Bossuyt, P.M.; Boutron, I.; Hoffmann, T.C.; Mulrow, C.D.; Shamseer, L.; Tetzlaff, J.M.; Akl, E.A.; Brennan, S.E.; et al. The PRISMA 2020 Statement: An Updated Guideline for Reporting Systematic Reviews. BMJ 2021, 372 , n71. [CrossRef] [PubMed]

51. Aslam, S.; Emmanuel, P. Formulating a Researchable Question: A Critical Step for Facilitating Good Clinical Research. Indian J. Sex. Transm. Dis. AIDS 2010, 31, 47-50. [CrossRef]

52. The Cochrane Collaboration. The Cochrane Highly Sensitive Search Strategies for Identifying Randomized trials in MEDLINE Available online: https://handbook-5-1.cochrane.org/chapter_6/6_4_11_1_the_cochrane_highly_sensitive_search_strategies_ for.htm (accessed on 1 September 2021).

53. Solomon, D.; Aderaw, Z.; Tegegne, T.K. Minimum Dietary Diversity and Associated Factors among Children Aged 6-23 Months in Addis Ababa, Ethiopia. Int. J. Equity Health 2017, 16, 181. [CrossRef]

54. Handu, D.; Moloney, L.; Wolfram, T.; Ziegler, P.; Acosta, A.; Steiber, A. Academy of Nutrition and Dietetics Methodology for Conducting Systematic Reviews for the Evidence Analysis Library. J. Acad. Nutr. Diet. 2016, 116, 311-318. [CrossRef]

55. Rauh-Pfeiffer, A.; Handel, U.; Demmelmair, H.; Peissner, W.; Niesser, M.; Moretti, D.; Martens, V.; Wiseman, S.; Weichert, J.; Heene, M.; et al. Three-Month B Vitamin Supplementation in Pre-School Children Affects Folate Status and Homocysteine, but Not Cognitive Performance. Eur. J. Nutr. 2014, 53, 1445-1456. [CrossRef]

56. Demmelmair, H.; Øyen, J.; Pickert, T.; Rauh-Pfeiffer, A.; Stormark, K.M.; Graff, I.E.; Lie, Ø.; Kjellevold, M.; Koletzko, B. The Effect of Atlantic Salmon Consumption on the Cognitive Performance of Preschool Children-A Randomized Controlled Trial. Clin. Nutr. 2019, 38, 2558-2568. [CrossRef]

57. Øyen, J.; Kvestad, I.; Midtbø, L.K.; Graff, I.E.; Hysing, M.; Stormark, K.M.; Markhus, M.W.; Baste, V.; Frøyland, L.; Koletzko, B.; et al. Fatty Fish Intake and Cognitive Function: FINS-KIDS, a Randomized Controlled Trial in Preschool Children. BMC Med. 2018, 16, 41. [CrossRef] [PubMed]

58. Metallinos-Katsaras, E.; Valassi-Adam, E.; Dewey, K.G.; Lönnerdal, B.; Stamoulakatou, A.; Pollitt, E. Effect of Iron Supplementation on Cognition in Greek Preschoolers. Eur. J. Clin. Nutr. 2004, 58, 1532-1542. [CrossRef] [PubMed]

59. Ryan, A.S.; Nelson, E.B. Assessing the Effect of Docosahexaenoic Acid on Cognitive Functions in Healthy, Preschool Children: A Randomized, Placebo-Controlled, Double-Blind Study. Clin. Pediatr. 2008, 47, 355-362. [CrossRef] [PubMed]

60. Kvestad, I.; Vabø, S.; Kjellevold, M.; Nøstbakken, O.J.; Midtbø, L.K.; Hysing, M.; Markhus, M.W.; Madsen, L.; Handeland, K.; Graff, I.E.; et al. Fatty Fish, Hair Mercury and Cognitive Function in Norwegian Preschool Children: Results from the Randomized Controlled Trial FINS-KIDS. Environ. Int. 2018, 121 Pt 2, 1098-1105. [CrossRef]

61. Aboud, F.E.; Bougma, K.; Lemma, T.; Marquis, G.S. Evaluation of the Effects of Iodized Salt on the Mental Development of Preschool-Aged Children: A Cluster Randomized Trial in Northern Ethiopia: Iodized Salt and Child Development in Ethiopia. Matern. Child. Nutr. 2017, 13, e12322. [CrossRef]

62. Roy Choudhury, D.; Nair Krishnapillai, M.; Nagalla, B.; Vijaya Kankipati, R.; Ghosh, S.; Buwade, J.; Fernandez-Rao, S. Guava with an Institutional Supplementary Meal Improves Iron Status of Preschoolers: A Cluster-Randomized Controlled Trial. Ann. N. Y. Acad. Sci. 2021, 1492, 82-95. [CrossRef]

63. Schneider, N.; Geiser, E.; Gosoniu, L.M.; Wibowo, Y.; Gentile-Rapinett, G.; Tedjasaputra, M.S.; Sastroasmoro, S. A Combined Dietary and Cognitive Intervention in 3-5-Year-Old Children in Indonesia: A Randomized Controlled Trial. Nutrients 2018, 10, 1394. [CrossRef]

64. Ogunlade, A.O.; Kruger, H.S.; Jerling, J.C.; Smuts, C.M.; Covic, N.; Hanekom, S.M.; Mamabolo, R.L.; Kvalsvig, J. Point-of-Use Micronutrient Fortification: Lessons Learned in Implementing a Preschool-Based Pilot Trial in South Africa. Int. J. Food Sci. Nutr. 2011, 62, 1-16. [CrossRef]

65. Black, M.M.; Fernandez-Rao, S.; Nair, K.M.; Balakrishna, N.; Tilton, N.; Radhakrishna, K.V.; Ravinder, P.; Harding, K.B.; Reinhart, G.; Yimgang, D.P.; et al. A Randomized Multiple Micronutrient Powder Point-of-Use Fortification Trial Implemented in Indian Preschools Increases Expressive Language and Reduces Anemia and Iron Deficiency. J. Nutr. 2021, 151, $2029-2042$. [CrossRef]

66. Roberts, S.B.; Franceschini, M.A.; Silver, R.E.; Taylor, S.F.; de Sa, A.B.; Có, R.; Sonco, A.; Krauss, A.; Taetzsch, A.; Webb, P.; et al. Effects of Food Supplementation on Cognitive Function, Cerebral Blood Flow, and Nutritional Status in Young Children at Risk of Undernutrition: Randomized Controlled Trial. BMJ 2020, 370, m2397. [CrossRef]

67. Masud Parvez, G.M.; Uzzaman, S.; Akanda, K.M.; Mehjabin, S. Open Access: Toxicology \& Research A short review on a Nutritional Fruit: Guava Research Article. Available online: https://www.biocoreopen.org/oatr/A-short-review-on-aNutritional-Fruit--Guava.pdf (accessed on 16 January 2022).

68. Hallberg, L.; Brune, M.; Rossander, L. The Role of Vitamin C in Iron Absorption. Int. J. Vitam. Nutr. Res. Suppl. 1989, 30, 103-108. [PubMed]

69. Sachdev, H.P.S.; Gera, T.; Nestel, P. Effect of Iron Supplementation on Mental and Motor Development in Children: Systematic Review of Randomised Controlled Trials. Public Health Nutr. 2005, 8, 117-132. [CrossRef] [PubMed] 
70. Lam, L.F.; Lawlis, T.R. Feeding the Brain-The Effects of Micronutrient Interventions on Cognitive Performance among SchoolAged Children: A Systematic Review of Randomized Controlled Trials. Clin. Nutr. 2017, 36, 1007-1014. [CrossRef] [PubMed]

71. World Health Organization. Guidelines on Food Fortification with Micronutrients. Available online: https://www.who.int/ publications/i/item/9241594012 (accessed on 1 August 2021). 